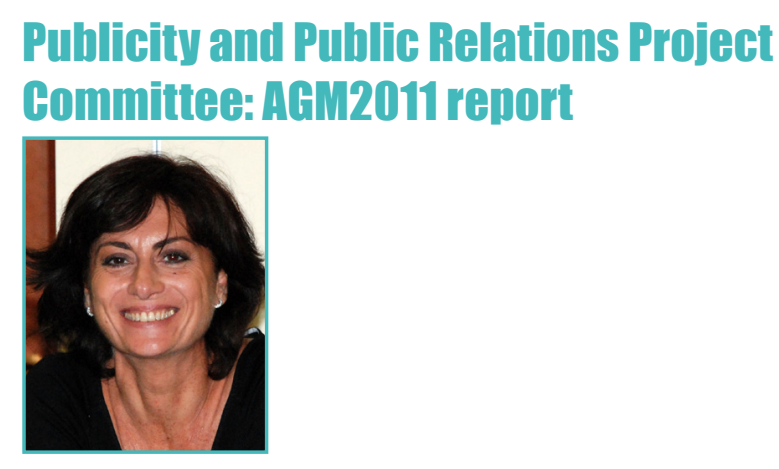

\section{Domenica D'Elia}

CNR-Institute for Biomedical Technologies, Bari, Italy

\section{Commitee members \\ Chair: Secretary: Member: Member: Member: Domenica D'Elia Lubos Klucar Martin Norling Judit Kumuthini Kanchana Senanayake}

The main mission of the P\&PR PC is to nurture and promote EMBnet's image at large. The P\&PR PC is responsible for promoting any type of EMBnet activities, for the advertisement of products and services provided by the EMBnet community, as well as for proposing and developing new strategies aiming to enhance EMBnet's visibility, and to take care of public relationships with EMBnet communities and related networks/societies. In this document, we report proposals, activities and achievements of the committee from June 2010 to May 2011.

\section{Introduction}

Since June 2010, the PC's composition and organisation has changed. Namely, Pedro Fernandes, who led the committee since its establishment, moved to guide the ET PC; Judit Kumuthini and Kanchana Senanayache, from the South African and Sri Lankan nodes respectively, joined the committee as new board members, while Goran Nerish, Andreas Gisel and Pedro Fernandes joined the committee as associate members. We would like to thank Pedro for the great contribution he gave in his role of chair of the committee up to June 2010, and for his continuous support as associate member.

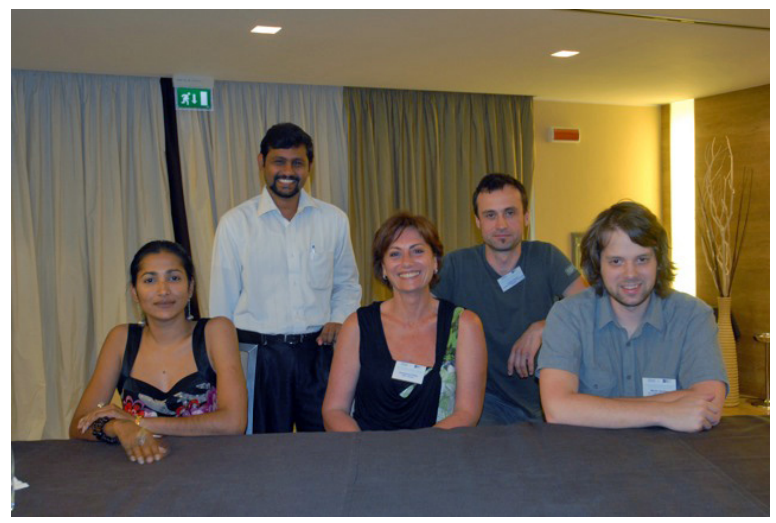

Figure 1. The Publicity and Public Relations Project Commitee (P\&PR PC) 2010. Left to Right: Judit Kumithini, Kanchana Senanayake, Domenica D'Elia, Lubos Klucar and Martin Norling.

\section{Committee meetings and documents}

A first meeting aiming to agree on the new structure and organisation of the P\&PR PC was held in June, immediately after the conclusion of the EMBnet AGM 2010 in Ruvo di Puglia (IT). The committee elected as chair Domenica D'Elia, and as secretary, Lubos Klucar. From July 2010 to May 2011, activity plan proposals and working organisation of the committee were discussed and agreed by e-mail and by three Virtual Meetings (VMs) (22 Sep., 2010; 21 Oct., 2010; 18 Jan., 2011). The P\&PR PC provided a business plan for the period 2010-2013 on October 2010, and released three activity reports as follow: Nov 2010-Jan 2011, Feb 2011 and March 2011. All these documents are available to EMBnet members from the new archive of the EMBnet website.

\section{P\&PR PC visions and goals}

\section{Assessment of EMBnet community identity and needs}

We propose the implementation of periodical surveys, at least on a yearly basis, aiming to get a snapshot of EMBnet and EMBnet communities (i.e., collaborating research groups in the same or different institutions). This activity would help to assess current EMBnet identity (bioinformatics research, capacity-building), developmental potential, as well as the needs of EMBnet-related communities (i.e., training support, development of bioinformatics tools, advice on infrastructure solutions, organisation of thematic workshops, 
help to initiate bioinformatics activities). Interview results would help to enhance:

- effectiveness of EMBnet activity projects;

- linkage between EMBnet and local research communities;

- collaborative initiatives/projects amongst EMBnet members and related/associated communities;

- improvement of EMBnet website content and of its scientific and social impact.

\section{Real-time monitoring of EMBnet's business} activity (BAM)

The website should be complemented with a good Web Analytics tool to allow real-time monitoring of EMBnet's target and performance in line with the EMBnet strategic road map. This would allow the EMBnet board to quickly address problems (if any), to make better-informed business decisions, and to take full advantage of emerging opportunities. The first step has already been done, and the whole embnet.org site is being monitored using the Google Analytics system, as of June 2010.

\section{Publicity policy for the EMBnet website and EMBnet.journal}

The proposal is to dedicate some space on the EMBnet website and EMBnet.journa/ portal to publicity banners for commercial companies with interests in bioinformatics and related research fields (e.g, IT, Biotechnology and Life Sciences Companies). Income should be invested in tasks of strategic importance for EMBnet, such as the website, EMBnet.journal, sponsorship at key conferences, or establishment of special prices or travel grants for young students to EMBnet conferences, workshops, etc.

\section{EMBnet sponsorship at key conferences} Sponsorship at key conferences represents a strategic task for enhancing EMBnet visibility. We propose to define terms and conditions for implementing special cooperation agreements with other (even dissimilar) networks and societies for mutual sponsorship of products and activities at key conferences. Steps along these lines have already been taken through EMBnet sponsorship of the 2011 ISCB Africa ASBCB Conference on Bioinformatics', in Cape Town. The effectiveness of this policy is demonstrated by special agreements established between ISCB and EMBnet

1 http://www.iscb.org/iscbafrica2011 on a collaborative program for education and training in Bioinformatics. A press release ${ }^{2}$ on this agreement is available from the EMBnet website.

\section{EMBnet Contact Registration}

In line with the main objectives of the committee to use new strategies to strengthen the network of EMBnet contacts, the P\&PR PC has proposed to establish a registration mechanism through which people interested in the activities of EMBnet can register and hence receive news and posting comments to EMBnet without any type of obligation. A contact registration form ${ }^{3}$ has been designed on February 2011 and activated on March 2011. Registrations and statistics are recorded and accessible to privileged users.

Alerts on this initiative have been included in EMBnet publications (brochure and 16.1 issue of EMBnet.journal), distributed at the ISCB Africa conference (9-11 March, 2011) and at the workshop organised by EMBnet in conjunction with the conference ${ }^{4}$ (7-8 March 2011). Contacts registered up to May 2011 are from Cameroon, Canada, Ghana, Greece, Indonesia, Kenya, Mexico, South Africa, Sweden, Tanzania, Tunisia, United Kingdom, USA, and Vietnam. As expected, the majority (28\%) come from South Africa, as a result of dissemination carried out at the 2011 Africa ISCB conference in Cape Town, but other contacts learned about this initiative through the Web. Comments posted by contacts confirm the need of students and young researchers to get training (Master, basic and advanced courses in bioinformatics), a general interest in collaboration on the development of bioinformatics tools, and a desire to participate in our conference. An EMBnet contact mailing list has been implemented by the TM PC on April 2011, but much more is necessary to cope with new needs arising from this initiative. The EMBnet website must have a "Contact" page, and help desks need to be activated and maintained to provide adequate services.

2 http://journal.embnet.org/index.php/embnetjournal/article/view/217/477

3 http://www.embnet.org/en/ContactRegistration

4 http://www.iscb.org/iscbafricall-program 


\section{EMBnet website: proposals and achievements}

The P\&PR PC has designed and submitted to the EB and TM PC a first proposal for the re-modelling of the EMBnet website on October 2010. A working plan for the coordination and implementation of new developments has been definitively agreed with the TM PC and EB on March 2011. Some of the changes proposed could be implemented only after TM PC has completed the upgrading and updating of the new server, others changes have been made and are:

1. EMBnet documents archive;

2. Public archive of EMBnet conferences and workshops (coll. with TM PC);

3. EMBnet.digest main menu block with hyperlink to the last released digest and to the archive;

4. Internal mailing lists page: this page contains mailing lists maintained by EMBnet, and a guide on how to use the lists (coll. with TM PC);

5. National, Specialist and Associate Nodes lists: the menu block containing information about member nodes has been recently reorganised with the addition of the Associate nodes section. Node information has been updated (where possible) immediately after the AGM in 2010. Some work is still necessary in order to correctly place Specialist and Associate nodes in the relevant lists, as well as to update these lists with new nodes currently missing (coll. with TM PC);

6. Contact Registration Form: public and available from the main menu.

\section{New EMBnet logo}

The need to design a new logo for EMBnet was raised by our new global dimension and goals for "21st Century EMBnet" largely discussed and agreed at the AGM in 2010. From 2010, EMBnet decided to adopt a new brand and strapline. The occasion of EMBnet sponsorship at the "2011 ISCB Africa ASBCB Conference on Bioinformatics" prompted the development of a new logo design that could better represent the spirit and essence of EMBnet in its new global dimension. The P\&PR PC has proposed that, from 2011, this new logo is officially approved by EMBnet for any use and purpose in place of the old one (where applicable). This proposal was submitted to the EMBnet Board during the VGM on April 2011, and

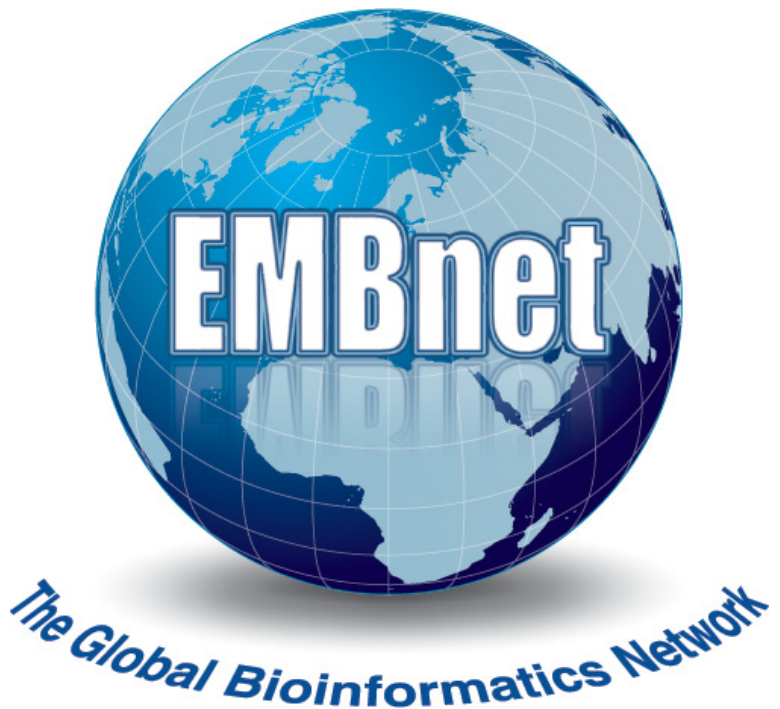

Figure 2. The new EMBnet logo.

will be voted on at forthcoming AGM (May 2011) in Portugal. 